\title{
Inversion of multiple data sets acquired by different array configuration of geoelectrical resistivity method
}

\section{Thong Duy Kieu*}

Faculty of Oil and Gas, Hanoi University of Mining and Geology, Vietnam

ARTICLE INFO ABSTRACT

Article history:

Received 15th Dec 2019

Revised $6^{\text {th }}$ Jan. 2020

Accepted 28 $8^{\text {th }}$ Feb. 2020

Keywords:

Inversion,

Resistivity,

Integration,

Electrode array.
The geoelectrical resistivity method is one of the most commonly used geophysical methods. This method uses differentelectrodes configuration, electrode array, depending on the purpose and conditions of the field, each type of array has its advantages and disadvantages. Due to the development of data acquisition technology, it is common for geoelectrical instruments enable to record data arising from different electrode arrays with negligible real-time construction. However, current software's only allows to process for each individual electrode array. Inverted models of different electrode array can be integrated to build a common earth model. However, due to the nature of the geophysical inversion is non-unique solutions, it means that there will be an infinite of models that can be suitable for a measurement in a certain noise level. This leads to the same measurement data in an area with different electrode array may produce different geoelectrical models making the dificulty for integration process. To solve this problem, we utilise the simultaneous joint inversion algorithm of data sets arising from multiple electrode arrays. The test results on synthetic data show that this combination is better than the solution of each individual electrode array. The best result is a combination of pole - dipole (PD), dipole - pole (DP) and dipole - dipole (DD).

Copyright (C) 2020 Hanoi University of Mining and Geology. All rights reserved.

\footnotetext{
${ }^{*}$ Corresponding author

E-mail: kieuduythong@humg.edu.vn

DOI: 10.46326/JMES.2020.61(1).06
} 


\section{Tạp chí Khoa học Kỹ thuật Mỏ - Địa chất}

Trang điện tử: http://tapchi.humg.edu.vn

\section{Giải ngược tổ hợp tài liệu của các hệ điện cực trong phương pháp điện trở suất 2D}

\section{Kiều Duy Thông*}

Khoa Dầu khí, Trường Đại học Mỏ - Địa chất, Việt Nam

\begin{tabular}{|c|c|}
\hline THÔNG TIN BÀI BÁO & TÓM TẮT \\
\hline $\begin{array}{l}\text { Quá trình: } \\
\text { Nhận bài 15/12/2019 } \\
\text { Sửa xong 06/01/2020 } \\
\text { Chấp nhận đăng 28/02/2020 } \\
\text { Tù̀ khóa: } \\
\text { Giải ngược, } \\
\text { Điện trở suất, } \\
\text { Tổ hợp, } \\
\text { Hệ điện cực. }\end{array}$ & 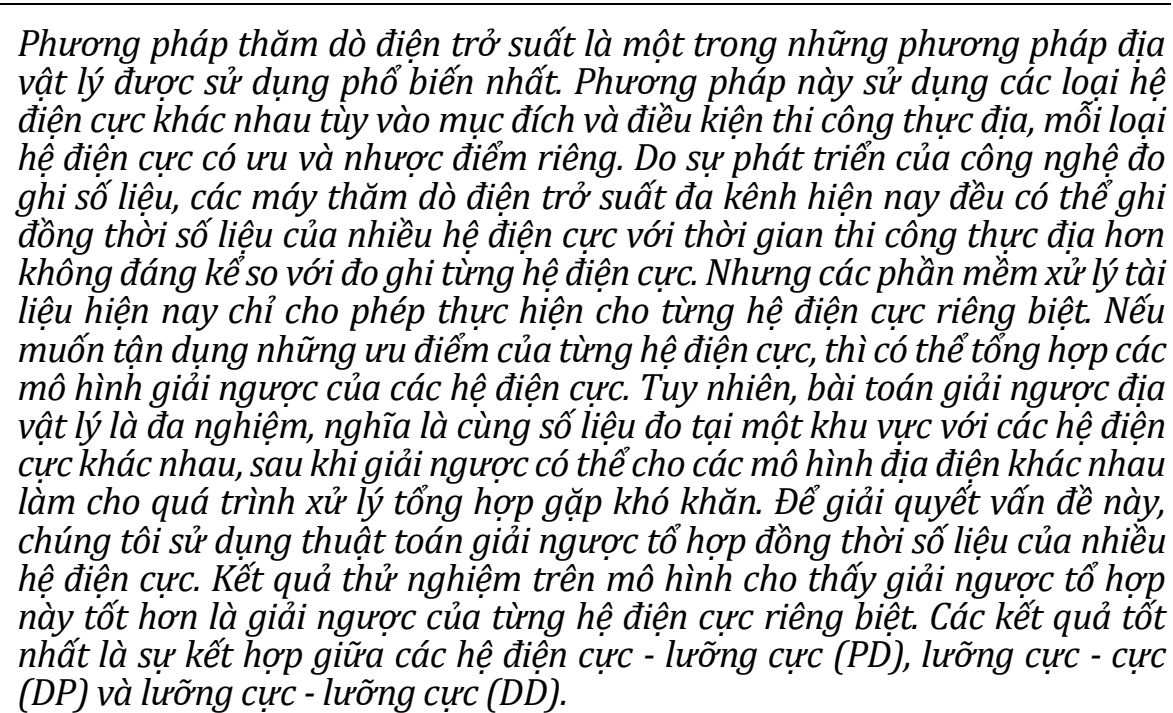 \\
\hline
\end{tabular}

C 2020 Trường Đại học Mỏ - Địa chất. Tất cả các quyền được bảo đảm.

\section{Mở đầu}

Địa vật lý (ĐVL) đã và đang là một công cụ rất hữu ích phục vụ cho các mục đích khác nhau. Điểm mạnh của các phương pháp ĐVL là khảo sát với nhiều tỉ lệ, không cần tiếp cận trực tiếp đối tượng. Tài liệu địa vật lý thường được xử lý để xây dựng mô hình phân bố tính chất vật lý liên quan đến đối

\footnotetext{
*Tác giả liên hệ

E - mail: kieuduythong@humg. edu.vn DOI: 10.46326/JMES.2020.61(1).06
}

tượng dưới mặt đất, quá trình này gọi là giải ngược tài liệu địa vật lý, trong bài báo này để ngắn gọn chúng tôi gọi tắt là giải ngược. Vấn đề nan giải nhất trong giải ngược đó là tính đa trị của bài toán. Có nghĩa là sẽ có vô số mô hình phân bố tính chất vật lý dưới mặt đất có thể giải thích cho số liệu đo trên mặt đất trong một khoảng nhiễu nhất định. Do vậy, quá trình giải ngược địa vật lý cần có thêm thông tin để hạn chế tính đa trị, hay làm hẹp miền nghiệm của bài toán. Một phương pháp hữu hiệu đang được sử dụng rộng rãi trên thế giới hiện nay là sử dụng tổ hợp tài liệu các phương pháp ĐVL để giải ngược (Gallardo and Meju, 2011; Heincke et 
al., 2017; Lines et al., 1988; Moorkamp, 2017; Moorkamp et al., 2011; Paasche and Tronicke, 2007; Sun and Li, 2015; Thông, 2018; Vozoff and Jupp, 1975a). Hơn nữa, độ phân giải của các phương địa vật lý là khác nhau, nên việc kết hợp các phương pháp trong một quá trình giải ngược tổ hợp cũng làm tăng độ phân giải của kết quả giải ngược (Vozoff and Jupp, 1975b).

Tổng quan về giải ngược tổ hợp đã được giới thiệu trong (Thông, 2018). Trong vấn đề giải ngược thì hai điểm quan trọng nhất cần phải giải quyết. Thứ nhất là tìm được mối liên hệ giữa các tham số vật lý của mô hình, các mô hình tham số vật lý của các phương pháp thành viên có thể liên hệ với nhau bằng mối liên kết cấu trúc (structural link) hoặc thạch học (petrohysical link) (Thông, 2018). Thứ hai là cách thức tiến hành giải ngược tổ hợp số liệu, hiện tại trên thế giới gồm hai cách thức chủ yếu (1) giải ngược đồng thời tài liệu địa vật lý (joint inversion); số liệu của các phương pháp ĐVL khác nhau được tiến hành trong cùng một quá trình. (2) Giải ngược lần lượt tài liệu địa vật lý (co-operative inversion). Giải ngược các phương pháp nối tiếp nhau, kết quả của quá trình giải ngược của phương pháp trước được đưa vào đầu vào cho quá trình giải ngược của phương pháp sau. Mỗi cách thức liên kết mô hình và quá trình giải ngược đều có ưu điểm và nhược điểm riêng và được áp dụng tùy theo bài toán cụ thể (Thông, 2018).

Trong nghiên cứu này, chúng tôi áp dụng giải ngược tổ hợp tài liệu địa vật lý cho bài toán điện trở suất 2D. Đây là phương pháp được sử dụng phổ biến hiện nay với các hệ điện cực thông dụng bao gồm: lưỡng cự - lưỡng cực (DD), cực - lưỡng cực (PD), lưỡng cực - cực (DP) và Wenner Schlumberger (WS) (Hình 1). Mỗi loại hệ điện cực có ưu và nhược điểm riêng (Athanasiou et al., 2007). Với sự phát triển của công nghệ đo nghi, các máy đo điện đa cực thường có khả năng đo liên tục các hệ điện cực khác nhau với chi phí về thời gian không nhiều. Do vậy, câu hỏi đặt ra là liệu khi xử lý giải ngược tổ hợp của các hệ điện cực này với nhau nhằm tận dụng ưu điểm của từng hệ điện cực có đem lại kết quả tốt hơn kết quả xử lý truyền thống từng hệ điện cực một hay không. Do đặc điểm của bài toán này là thực hiện trên cùng phương pháp điện trở suất nên chúng tôi áp dụng giải ngược tổ hợp đồng thời (joint inversion). Đã có các nghiên cứu được thực hiện về vấn đề này
(Athanasiou et al., 2007), tuy nhiên trong các nghiên cứu này các tác giả đánh giá dựa trên mô hình rất đơn giản, mô hình chỉ gồm các khối đồng nhất và không tính đến ảnh hưởng của bất đồng nhất của lớp trên cùng. Trong nghiên cứu này chúng tôi sử dụng mô hình gần với thực tế hơn khi tính đến bất đồng nhất gần mặt đất. Tổ hợp các hệ điện cực được sử dụng cũng khác so với nghiên cứu của nghiên cứu của Athanasiou et al., (2007).

\section{Giải ngược tài liệu địa vật lý}

\subsection{Lý thuyết cơ bản về giải ngược}

Giải ngược tài liệu ĐVL là quá trình xác định sự phân bố tính chất vật lý trong không gian dưới mặt đất từ tài liệu đo được. Thông thường hiện nay giải ngược được thực hiện bởi quá trình giải lặp, cực tiểu hóa hàm sau:

$$
\emptyset=\emptyset_{d}+\beta \emptyset_{m}
$$

Trong đó: $\phi_{d}$ - công thức (2), là độ lệch (misfit) hay sai số (error) giữa tài liệu đo được và tài liệu lý thuyết tính toán từ mô hình; $\phi_{m}$ - công thức (3), đánh giá tham số mô hình, có thể là độ trơn (smoothness) như trong nghiên cứu của Constable et al. (1987), hoặc bao gồm thêm các kỹ thuật hạn chế nghiệm khác như trong các công trình của Menke (2015); Sun and Li $(2014,2015)$; Tarantola and Valette (1982); $\beta$ - (Lagarian regulerization parameter) là tham số cân bằng giữa sai số và tham số cấu trúc mô hình (Tikhonov and Arsenin, 1977)

$$
\begin{gathered}
\emptyset_{d}=\|W d-W f(m)\|_{2}^{2} \\
\emptyset_{m}=\|\tilde{\partial} m\|_{2}^{2}
\end{gathered}
$$

Trong đó $\|\quad\|_{2}$ - tiêu chuẩn Euclidian, $d=\left(d_{1}\right.$, $\left.d_{2}, \ldots, d_{M}\right)^{\mathrm{T}}$ và $m=\left(\mathrm{m}_{1}, m_{2}, \ldots, m_{N}\right)^{\mathrm{T}}$ là số liệu và tham số mô hình, $\mathrm{f}(\mathrm{m})$ - toán tử mô hình hóa, trong địa vật lý toán tử này thường là hàm phi tuyến; $W$ là ma trận đường chéo $N x N$ phương sai của số liệu $\sigma(4)$, và $\tilde{\partial}$ là toán tử hiệu (Different operator).

$$
W=\operatorname{diag}\left\{1 / \sigma_{1}, 1 / \sigma_{2}, \ldots, 1 / \sigma_{N}\right\},
$$

Để giải bài toán giải ngược với hàm phi tuyến trong địa vật lý, thuật toán thường được sử dụng là giải lặp (Constable et al., 1987). Hàm $f(m)$ có thể được xấp xỉ thành chuỗi Taylor như sau: 


$$
f\left(m_{(1)}+\Delta m\right)=f\left(m_{(1)}\right)+J_{(1)} \Delta m
$$

Trong đó $\Delta m=m_{(2)}-m_{(1)}$ là véc tơ thể hiện sự thay đổi của các tham số mô hình, gọi tắt là véc tơ biến đổi mô hình, và $\mathrm{J}_{(1)}$ là ma trận đạo hàm bán phần của hàm $f(m)$ theo vec tơ tham số mô hình $m_{(1)}$, Jacobian. có:

Từ các phương trình (1), (2) (3), (4) và (5) ta

$$
\emptyset=\left\|W d_{(1)}-W J_{(1)} m_{(2)}\right\|_{2}^{2}+\beta\left\|\tilde{\partial} m_{(2)}\right\|_{2}^{2}
$$

Trong đó

$$
d_{(1)}=d-f\left(m_{(1)}\right)+J_{(1)} m_{(1)} .
$$

Đạo hàm hàm số (6) theo tham số của mô hình $m_{(2)}:$

$$
\frac{\partial \emptyset}{\partial m_{(2)}}=2\left[\left(_{(1)}{ }^{T} A J_{(1)}+\beta H\right] m_{(2)}-2 J_{(1)}{ }^{T} A d_{(1)}^{(8)}\right.
$$

Trong đó: $H=\widetilde{\partial}^{T} \widetilde{\partial}$ và $A=W^{T} W$, rồi cho đạo hàm này bằng không, ta có thể tìm được véc tơ tham số mô hình thỏa mãn điều kiện phiếm hàm (6) được cực tiểu hóa. Sau một vài phép biến đổi ta sẽ tìm được véc tơ biến đổi mô hình sau bước lặp thứ $i(9)$.

$$
\Delta m_{(i+1)}=\left[U_{(i)}{ }^{T} A J_{(i)}+\beta H\right]^{-1}\left[J_{(i)}{ }^{T} A \Delta d_{(i)}\right]
$$

Trong đó $\Delta d_{(i)}=d-f\left(m_{(i)}\right)$ là sai số giữa số liệu thực tế đo được và số liệu lý thuyết tình từ mô hình với tham số $m_{(i)}$.

\subsection{Giải ngược tổ hợp tài liệu điện trở suất tù̀ các hệ điện cực}

Phương pháp điện trở suất sử dụng rất nhiều hệ điện cực khác nhau (Szalai and Szarka, 2008). Mỗi loại hệ điện cực có ưu điểm và nhược điểm riêng, tùy thuộc vào mô hình địa điện (Athanasiou et al., 2007; Dahlin and Zhou, 2004, 2006; Szalai et

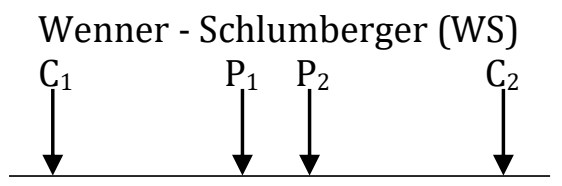

Dipole - Dipole (DD)

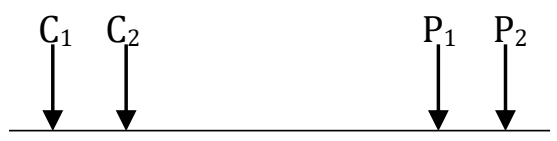

al., 2011). Trên thực tế phương pháp điện trở suất thường sử dụng các hệ điện cực được mô tả trong Hình 1. Trong trường hợp tổng quát, hệ điện cực WS thường có độ phân giải đứng tốt, ngược lại độ phân giải ngang của các hệ điện cực $\mathrm{DD}, \mathrm{PD}$ và $\mathrm{DP}$ tốt hơn (Dahlin and Zhou, 2004). Chiều sâu nghiên cứu của hệ điện cực PD hoặc DP tốt hơn hệ điện cực DD và hệ điện cực WS (Szalai et al., 2011). Tuy nhiên, ảnh hưởng của các bất đồng nhất địa phương gần mặt đất thường không liên quan đến đối tường nghiên cứu lại tăng dần từ hệ điện cực WS, đến PD hoặc DP và cao nhất đối với hệ điện cực DD (Dahlin and Zhou, 2004). Do vậy, giải ngược tổ hợp tài liệu của các hệ điện cực có thể cho kết quả tốt hơn giải ngược tài liệu của từng hệ điện cực riêng lẻ, vì có thể tận dụng ưu điểm của hệ điện cực này để bù đắp nhược điểm của hệ điện cực kia.

Do điện trở suất biểu kiến của cùng một mô hình địa điện sẽ phụ thuộc vào hệ điện cực sử dụng. Để tránh việc giá trị điện trở suất biểu kiến của hệ điện cực này chiếm vai trò quyết định trong quá trình giải ngược tổ hợp. Ví dụ trong trường hợp hệ điện cực DD thường có ảnh hưởng quyết định đến quá trình giải ngược tổ hợp (Athanasiou et al., 2007), nên phải thiết lập một hệ số cân bằng. Trong công trình của Athanasiou et al. (2007), các tác giả dựa vào độ nhạy mô hình, hay ma trận Jacobian để tính hệ số này. Kết quả của công trình này cho thấy, rất khó để đánh giá hiệu quả sử dụng hệ số cân bằng. Chúng tôi cho rằng nguyên nhân có thể là do việc tính hệ số này theo độ nhạy mô hình là không phù hợp, vì bản chất của hệ số cân bằng này phải liên quan đến điện trở suất biểu kiến. Do vậy, trong nghiên cứu này chúng tôi sử dụng cách tính hệ số cân bằng dựa vào điện trở suất biểu kiến, kỹ thuật này được đề xuất bởi Vozoff and Jupp (1975b).

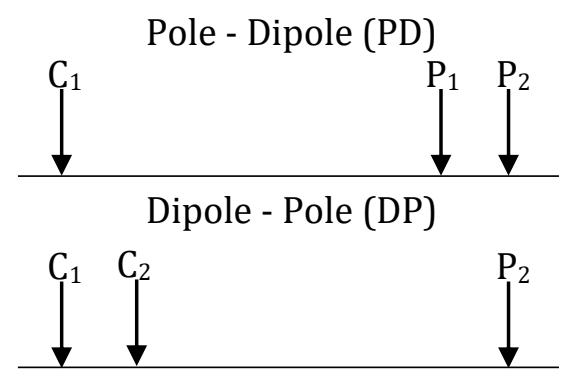

Hình 1: Các hệ điện cực thường dùng: Wenner - Schlumberger (WS), lưỡng cực trục (DD), ba cực thuận $(P D)$, và ba cực nghịch (DP). 


\section{Kết quả và thảo luận}

\subsection{Mô hình}

Trong nghiên cứu này chúng tôi thiết lập một mô hình lý thuyết (Hình 2). Giá trị điện trở suất của mô hình này được chia làm 4 nhóm và được tính như trong Bảng 1 . Có thể thấy 4 nhóm điện trở bao gồm nhóm điện trở suất thấp $\left(\operatorname{Res}_{1}\right)$, nhóm điện trở suất trung bình $\left(\operatorname{Res}_{2}\right)$, nhóm điện trở suất cao $\left(\operatorname{Res}_{3}\right)$ và nhóm điện trở suất rất cao $\left(\operatorname{Res}_{4}\right)$. Trong mô hình này (Hình 1$)$, giá trị của các khối trong mô hình được sắp xếp ngẫu nhiên. Lớp trên cùng gồm giá trị điện trở suất của hai nhóm $\operatorname{Res}_{2}$ và $\operatorname{Res}_{3}$. Lớp này đặc trưng cho lớp phủ, thường có có giá điện trở suất thay đổi mạnh. Lớp thứ hai có giá trị điện trở suất của nhóm $\operatorname{Res}_{2}$, và lớp cuối cùng có giá trị điện trở suất của nhóm Res $_{3}$. Hai khối bất đồng nhất địa phương $O_{1}$ và $O_{2}$ có giá trị điện trở suất thấp $\operatorname{Res}_{1}$ và rất cao $\operatorname{Res}_{4}$. Số liệu tính từ mô hình này được cộng thêm phần nhiễu ngẫu nhiên, $5 \%$ giá trị điện trở suất biểu kiến, cho số liệu mô phỏng gần với thực tế.
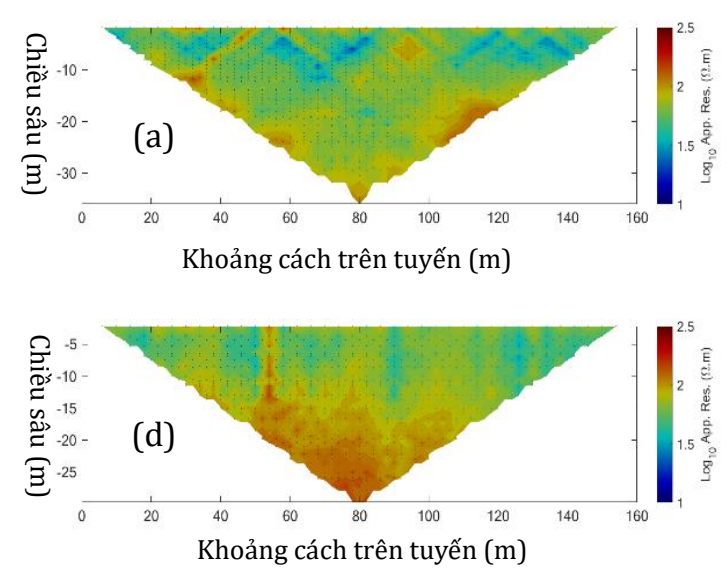

Bảng 1. Giá trị điện trở suất trong mô hình (Hình 2). Trong đó rand là hàm ngẫu nhiên. Giá trị điện trở suất của 4 nhóm được ký hiệu Res $1, \operatorname{Res}_{2}, \operatorname{Res}_{3}$, và Res4.

\begin{tabular}{|c|c|c|}
\hline Nhóm & Công thức tính & Giá trị điện trở suất $(\Omega \mathrm{m})$ \\
\hline \multirow{4}{*}{1} & \multirow{4}{*}{$\begin{array}{l}\operatorname{Res}_{1} \\
=10^{\left[\frac{\text { and }}{5}-0.5\right]}\end{array}$} & \begin{tabular}{|c|}
1.01 \\
\end{tabular} \\
\hline & & 1.07 \\
\hline & & 1.29 \\
\hline & & 1.08 \\
\hline \multirow{4}{*}{2} & \multirow{4}{*}{$\begin{array}{l}\operatorname{Res}_{2} \\
=10^{\left[\frac{\text { rand }}{5}+1\right]}\end{array}$} & 45.21 \\
\hline & & 42.15 \\
\hline & & 46.24 \\
\hline & & 31.25 \\
\hline \multirow{4}{*}{3} & \multirow{4}{*}{$\begin{array}{l}\operatorname{Res}_{3} \\
=10^{\left[\frac{\text { rand }}{5}+2\right]}\end{array}$} & 314.43 \\
\hline & & 339.80 \\
\hline & & 473.00 \\
\hline & & 326.74 \\
\hline \multirow{4}{*}{4} & \multirow{4}{*}{$\begin{array}{l}\operatorname{Res}_{4} \\
=10^{\left[\frac{\mathrm{rand}}{5}+3.1\right]}\end{array}$} & 1622.43 \\
\hline & & 1371.76 \\
\hline & & 1941.15 \\
\hline & & 1973.72 \\
\hline
\end{tabular}
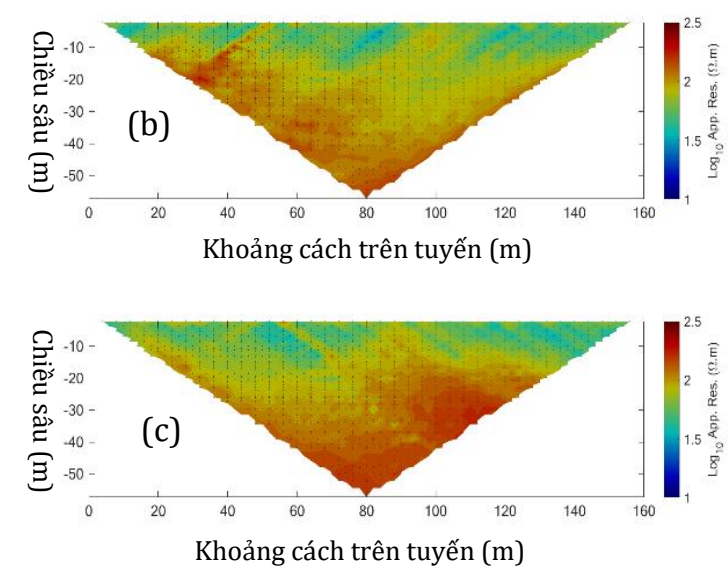

Điện trở suất $(\Omega \mathrm{m})$

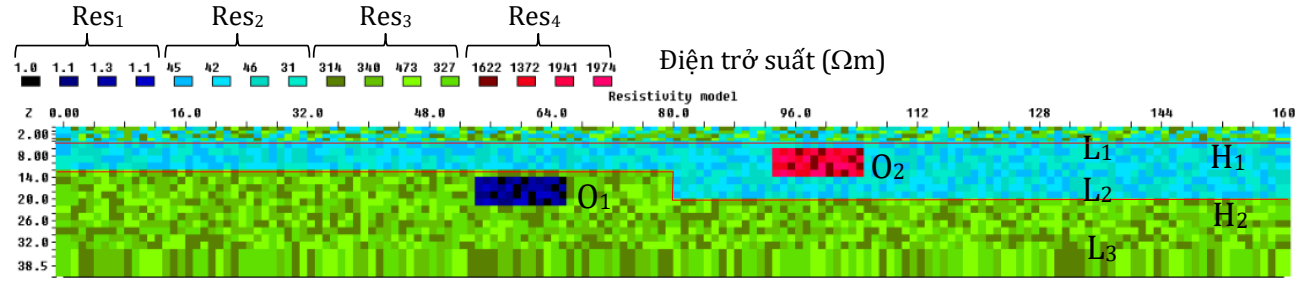

Hình 2. Mô hình địa điện (e) bao gồm ba lớp $L_{1}, L_{2}$ và $L_{3}$ được phân chia bởi hai ranh giới $H_{1}$ và $H_{2}$ (các đường màu đỏ), có hai khối bất đồng nhất địa phương $O_{1}$ và $O_{2}$. Giá trị điện trở suất mô hình thể hiện trong Bảng 1. Giá trị điện trở suất biểu kiến tính từ mô hình cộng với $5 \%$ nhiễu ngẫu nhiên bởi các hệ điện cực DD (a), PD (b), DP (c) và WS (d) được thực hiện bằng phần mềm Res 2 dmod [23]. Khoảng cách giữa hai điện cực liên tiếp là $4.0 \mathrm{~m}$. Trong các hình (a), (b), (c) và (d) dải màu thể hiện giá trị logarit cơ số 10 điện trở suất biểu kiến; các chấm màu đen thể hiện vị trí điểm lấy số liệu. 
Trong mô hình này việc sử dụng tài liệu đo điện trở suất để xác định các ranh giới $H_{1}$ và $H_{2}$ là tương đối dễ ràng, tuy nhiên việc xác định các đối tượng $O_{1}$ và $O_{2}$ là rất khó khăn, nhất là $O_{1}$. Thứ nhất là ảnh hưởng bất đồng nhất lớp trên cùng $\mathrm{L}_{1}$. Thứ hai, đối tượng $\mathrm{O}_{1}$ tuy có điện trở suất khác biệt lớn so với môi trường vây quanh, nhưng do nằm ở chiều sâu lớn và nằm ngay dưới lớp $L_{2}$ có điện trở suất thấp. Do vậy, một trong những điểm quan trọng nhất trong bài toán này là đánh giá xem liệu các hệ điện cực phát hiện các đối tượng $O_{1}$ và $O_{2}$ như thế nào.

\subsection{Kết quả giải ngược}

\subsubsection{Kết quả giải ngược cho tùng hệ điện cực}

Để kiểm chứng hiệu quả của việc kết hợp các hệ điện cực, đầu tiên chúng tôi chạy giải ngược cho từng hệ điện cực, sử dụng phần mềm đang được sử dụng rộng rãi trên thế giới, Res 2 dinv. Kết quả được thể hiện trên Hình 3. Các kết quả này cho thấy nhìn chung các ranh giới $H_{1}$ và $H_{2}$ phân chia giữa các lớp $L_{1}, L_{2}$ và $L_{3}$ được thể hiện trên các mô hình giải ngược của cả bốn hệ điện cực. Đối tượng $\mathrm{O}_{2}$ có thể nhìn thấy trên các mô hình này, nhưng đối tượng $O_{1}$ trong tất cả các trường hợp đều
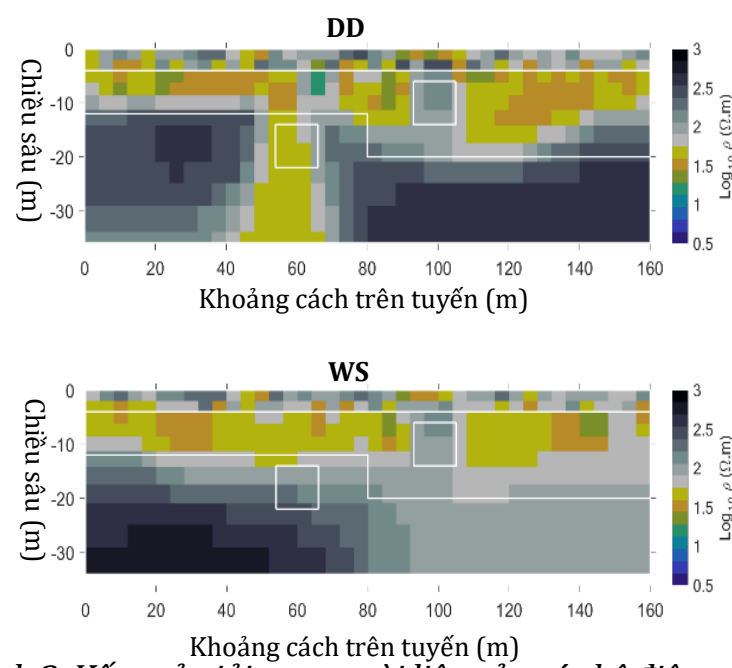

không rõ ràng. Đối tượng $O_{1}$ này tạo thành một đới dị thường điện trở suất thấp ở các kết quả của hệ điện cực $\mathrm{DD}, \mathrm{PD}$ và $\mathrm{DP}$ giống như dị thường của đới đứt gãy, thậm chí $O_{1}$ không thấy thể hiện trong kết quả của hệ điện cực WS.

\subsubsection{Kết quả giải ngược tổ hợp}

Các kết quả giải ngược này được thực hiện bằng chương trình Matlab. Chúng tôi đã thay đổi chương trình từ chương trình gốc được công bố bởi Akca (2016) cho các quá trình giải ngược của bài báo này. Tổ hợp số liệu của các hệ điện cực được trình bày trong Bảng 2 . Trên Hình 4 thể hiện độ lệch (misfit) giữa số liệu tính toán lý thuyết và tài liệu đo thực tế (ở đây là tài liệu tính theo mô hình cộng với nhiễu ngẫu nhiên). Giá trị độ lệch càng nhỏ thể hiện số liệu tính lý thuyết và số liệu đo càng gần nhau. Độ lệch này suy giảm một cách ổn định theo các bước lặp thể hiện chương trình giải ngược làm việc tốt. Nhìn chung sự khác biệt độ lệch giữa các tổ hợp số liệu của điện cực là không nhiều và đều nằm trong mức nhiễu $5 \%$. Cần lưu ý là độ lệch nhỏ hơn không đảm bảo kết quả giải ngược tốt hơn, mà chỉ cần độ lệch này tương đương mức nhiễu là được (Constable et al., 2015).
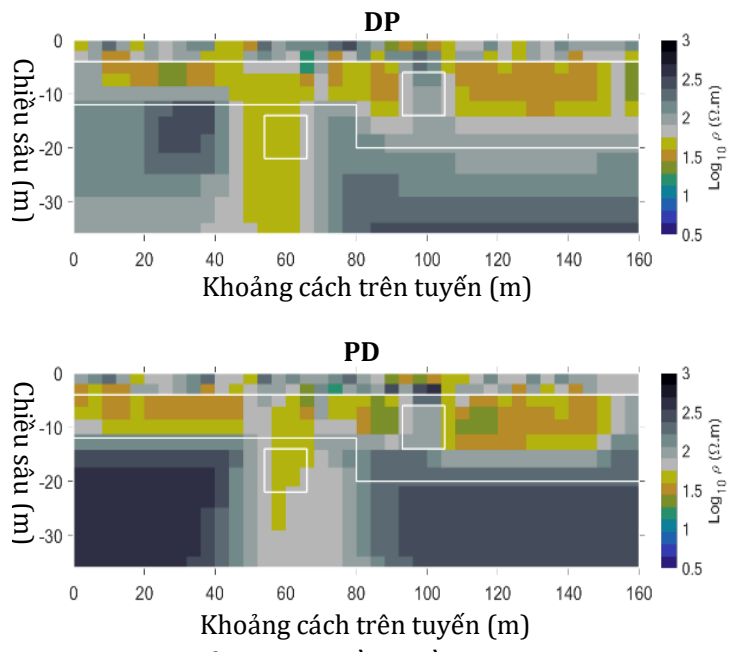

Hình 3. Kết quả giải ngược tài liệu của các hệ điện cực khác nhau, sủ dụng phần mềm Res 2 dinv [23]. Các đường màu trắng thể hiện vị trí của ranh giới $H_{1}$ và $H_{2}$ và hai đối tượng địa phương $O_{1}$ và $O_{2}$.

Bảng 2. Ký hiệu các tổ hợp số liệu từ các hệ điện cực khác nhau. Luu ý viết tắt của các hệ điện cực xem trong Hình 1.

\begin{tabular}{|c|c|c|c|c|c|}
\hline TT & Ký hiệu & Tổ hợp số liệu của các hệ điện cực & TT & Ký hiệu & Tổ hợp số liệu của các hệ điện cực \\
\hline 1 & PD-DD & PD và DD & 5 & PD-DP-DD & PD; DP; và DD \\
\hline 2 & PD-DP & PD và DP & 6 & PD-DP-WS & PD; DP và WS \\
\hline 3 & PD-WS & PD và WS & 7 & PD-DD-WS & PD; DD; và WS \\
\hline 4 & WS-DD & WS và DD & 8 & PD-DP-DD-WS & PD; DP; DD; và WS \\
\hline
\end{tabular}


Các mô hình giải ngược của các tổ hợp số liệu được thể hiện trên Hình 5 . Có thể rút ra một số nhận xét sau về các kết quả này. Thứ nhất, nếu so với kết quả giải ngược tài liệu của các hệ điện cực riêng rẽ thì các kết quả tổ hợp nhìn chung là tốt hơn. Lưu ý là thang màu của hai kết quả giải ngược trên Hình 3 và 5 được đặt giống nhau. Ngoài hai ranh giới $\mathrm{H}_{1}$ và $\mathrm{H}_{2}$ là các đối tượng dễ xác định, các đối tượng địa phương $\mathrm{O}_{1}$ và $\mathrm{O}_{2}$ được xác định trong quá trình giải ngược tổ hợp các điện cực là tốt hơn so với sử dụng riêng từng hệ điện cực. Thứ 2, các tổ hợp có sử dụng hệ điện cực WS cho kết quả không tốt bằng các hệ điện cực còn lại. Tổ hợp giữa hệ điện cực $\mathrm{PD}$ và $\mathrm{DD}$ hoặc $\mathrm{PD}$ và $\mathrm{DP}$ cho kết quả rất tốt, nhưng kết quả giải ngược tốt hơn với tổ hợp PD, DP và DD. Điều này cho thấy các kết quả của Athanasiou et al. (2007) thực hiện tổ hợp hai hệ điện cực WS với DD hoặc WS với PD chưa thực sự hiệu quả. Đây cũng là sự khác biệt và đóng góp quan trọng nhất của nghiên cứu của chúng tôi so với nghiên cứu đã được công bố.

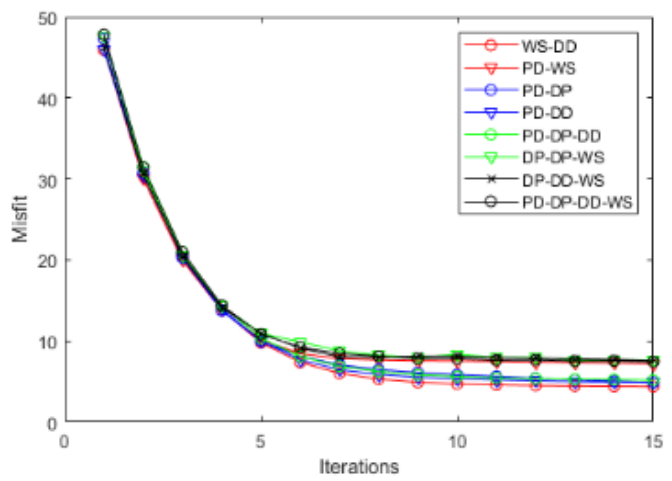

Hình 4. Độ lệch (misfit) giũa tài liệu đo và tài liệu tính lý thuyết thay đổi theo bước lặp (Iterations).
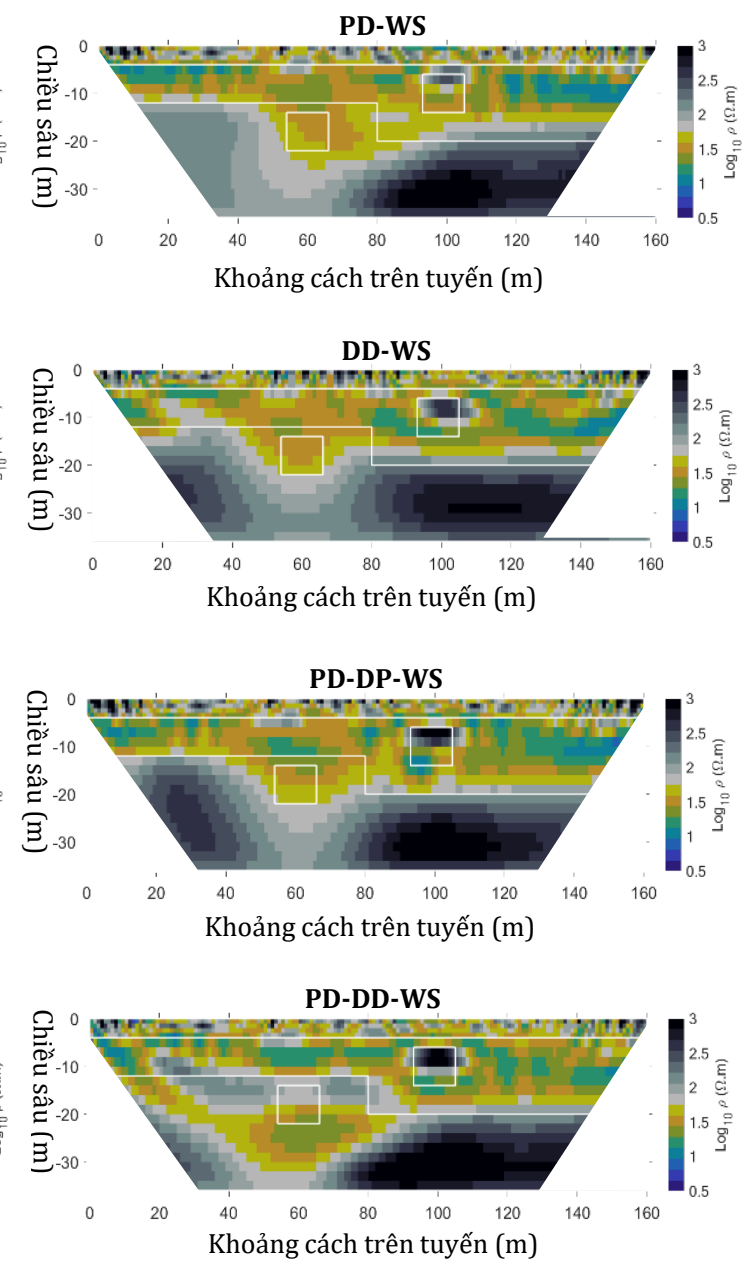

Hình 5. Kết quả giải ngược tổ hợp tài liệu của các hệ điện cực khác nhau (Bảng 2), sử dụng chương trình Matlab. Kết quả tổ hợp PD-DP-DD hoặc PD-DP-DD-WS là tốt nhất. Các đường màu trắng thể hiện vị trí của ranh giới $H_{1}$ và $H_{2}$ và hai đối tượng địa phương $O_{1}$ và $O_{2}$. 


\section{Kết luận}

Kết quả từ mô hình lý thuyết cho thấy giải ngược tổ hợp số liệu của các hệ điện cực cho mô hình tốt hơn so với mô hình của từng hệ điện cực riêng lẻ. Sử dụng tổ hợp số liệu các hệ điện cực đòi hỏi chi phí thực địa và tính toán cao hơn. Tuy nhiên, đây không phải là vấn đề lớn nhờ sự phát triển của công nghệ đo đạc và sức mạnh của các máy tính hiện nay. Kết quả này chỉ ra rằng tổ hợp giữa hệ điện cực: cực - lưỡng cực (PD), lưỡng cực - cực (DP) và lưỡng cực - lưỡng cực (DD) với mô hình dạng này cho kết quả tốt nhất. Trong nghiên cứu này chúng tôi mới tiến hành thử nghiệm trên tài liệu lý thuyết, dù cho đã được mô phỏng rất gần với điều kiện thực tế. Trong các nghiên cứu tiếp theo chúng tôi sẽ thử nghiệm trên tài liệu đo thực tế để đánh giá chính xác hơn khả năng áp dụng vào thực tế sản xuất của phương pháp này.

\section{Lời cảm ơn}

Bài báo này hoàn thành là kết quả của đề tài cấp cơ sở Trường Đại học Mỏ - Địa chất, mã số T18-36. Bản quyền phần mềm Matlab được tài trợ bởi công ty RoqSense. Chúng tôi chân thành cảm ơn Akca tác giả của bài báo "ELRIS2D: A MATLAB Package for the 2D Inversion of DC Resistivity/IP Data" đã cung cấp mã nguồn cho chương trình giải ngược tài liệu điện trở hai chiều. Chúng tôi trân trọng cảm ơn tiến sỹ Loke, người đã phát triển các phần mềm $\operatorname{Res}_{2}$ dmod và Res ${ }_{2}$ dinv và cung cấp các bản dùng miễn phí với mục đích nghiên cứu.

\section{Tài liệu tham khảo}

Akca, I., (2016). ELRIS2D: A MATLAB Package for the 2D Inversion of DC Resistivity/IP Data, Acta Geophysica 64. 443.

Athanasiou, E. N., Tsourlos, P. I., Papazachos, C. B., and Tsokas, G. N., (2007). Combined weighted inversion of electrical resistivity data arising from different array types: Journal of Applied Geophysics 62(2). 124 - 140.

Constable, S. C., Parker, R. L., and Constable, C. G., (1987). Occam's inversion; a practical algorithm for generating smooth models from electromagnetic sounding data: Geophysics 52(3) $3.289-300$.

Constable, S., Orange, A., and Key, K., (2015). And the geophysicist replied: "Which model do you want?": Geophysics 80(3). E(197-E212).

Dahlin, T., and Zhou, B., (2004). A numerical comparison of $2 \mathrm{D}$ resistivity imaging with 10 electrode arrays: Geophysical Prospecting, v. 52, no. 5, p. 379-398.-, (2006), Multiplegradient array measurements for multichannel 2D resistivity imaging: Near Surface Geophysics 4(2). 113 - 123.

Gallardo, L. A., and Meju, M. A., (2011). Structurecoupled multiphysics imaging in geophysical sciences: Reviews of Geophysics 49(1).

Heincke, B., Jegen, M., Moorkamp, M., Hobbs, R. W., and Chen, J., (2017). An adaptive coupling strategy for joint inversions that use petrophysical information as constraints: Journal of Applied Geophysics 136. 279 - 297.

Lines, L., Schultz, A., and Treitel, S., (1988). Cooperative inversion of geophysical data: Geophysics 53(1). 8 - 20.

Menke, W., (2015). Review of the Generalized Least Squares Method: Surveys in Geophysics 36(1). 1 - 25.

Moorkamp, M., (2017). Integrating Electromagnetic Data with Other Geophysical Observations for Enhanced Imaging of the Earth: A Tutorial and Review: Surveys in Geophysics.

Moorkamp, M., Heincke, B., Jegen, M., Roberts, A. W., and Hobbs, R. W., (2011). A framework for 3-D joint inversion of MT, gravity and seismic refraction data: Geophysical Journal International 184(1). 477 - 493.

Paasche, H., and Tronicke, J., (2007). Cooperative inversion of 2D geophysical data sets: a zonal approach based on fuzzy c-means cluster analysis: Geophysics 72(3). 35 - 39.

Sun, J., and Li, Y., (2014). Adaptive Lp inversion for simultaneous recovery of both blocky and smooth features in a geophysical model: Geophysical Journal International 197(2). 882 899; (2015), Multidomain petrophysically constrained inversion and geology differentiation using guided fuzzy c-means clustering: Geophysics 80(4). ID1-ID18.

Szalai, S., and Szarka, L., (2008). On the classification of surface geoelectric arrays: 
Geophysical Prospecting 56(2). 159 - 175.

Szalai, S., Novák, A., and Szarka, L., (2011). Which geoelectric array sees the deepest in a noisy environment? Depth of detectability values of multielectrode systems for various twodimensional models: Physics and Chemistry of the Earth, Parts A/B/C 36(16). 1398 - 1404.

Tarantola, A., and Valette, B., (1982). Generalized nonlinear inverse problems solved using the least squares criterion: Reviews of Geophysics 20(2). 19 - 232.
Tikhonov, A. N., and Arsenin, V. I. A., (1977). Solutions of ill-posed problems, Winston.

Thông, K. D., (2018). Tổng quan về giải ngược tổ hợp tài liệu địa vật lý, Hội nghị toàn quốc về khoa học trái đất và phát triển bền vững (ERSD). Trường Đại học Mỏ - Địa chất.

Vozoff, K., and Jupp, D. L. B., (1975a). Joint Inversion of Geophysical Data: Geophysical Journal of the Royal Astronomical Society, v. 42, no. 3, p. 977-991.-, (1975b, Joint Inversion of Geophysical Data 42(3). 977 - 991. 